\title{
Überleitung in die außerklinische invasive Beatmung
}

\author{
Jens Callegari, Wolfram Windisch, Jan Hendrik Storre
}

Eine außerklinische Beatmung nach erfolgloser Entwöhnung kann die Lebensqualität der Patienten steigern und auch - obwohl personell und finanziell aufwendig - letztlich Kosten sparen. Allerdings müssen dazu zahlreiche Voraussetzungen erfüllt sein.

\section{Einleitung}

Der medizinische Fortschritt hat in den letzten Jahren zu einer steigenden Zahl invasiv und nicht invasiv beatmeter Patienten geführt. Ein Teil dieser Patienten ist nach einem akuten respiratorischen Versagen oder aufgrund einer chronisch fortschreitenden Grunderkrankung dauerhaft nicht von der Beatmung entwöhnbar, sodass eine Beatmung außerhalb der Klinik notwendig wird. Grundsätzlich unterscheidet man bei der außerklinischen Beatmung zwischen invasiver und nicht invasiver Beatmung.

Epidemiologie. Epidemiologische Daten über die außerklinische Beatmung - insbesondere hinsichtlich der Inzidenz und Prävalenz - sind schwierig zu interpretieren. In einer aktuellen polnischen Studie wurde über einen 100-fachen Anstieg der außerklinisch beatmeten Patienten im Zeitraum von 2000 bis 2010 berichtet, wobei $50 \%$ davon invasiv beatmet waren [1]. Lloyd-Owen et al. fanden 2001 in einer europaweiten Umfrage eine Prävalenz von 6,6 außerklinisch beatmeten Patienten pro 100000 Einwohner mit einem Anteil von $13 \%$ invasiv beatmeter Patienten [2]. Aktuelle australische Daten berichten von lediglich 3\% über ein Tracheostoma beatmeten Patienten [3]. Eine kanadische Umfrage von Rose et al. zeigte, dass in Kanada mit
73\% ein Großteil der Patienten nicht invasiv beatmet wird [4]. Aktuelle deutsche Daten liegen derzeit nicht vor.

Insbesondere die Etablierung einer invasiven außerklinischen Beatmung ist eine große personelle, logistische und finanzielle Herausforderung. Dabei kommt der wachsenden Zahl nationaler Beatmungs- und Weaningzentren eine bedeutende Rolle hinsichtlich der Auswahl und Evaluation der Patienten sowie der Planung und Organisation der Überleitung in die außerklinische Beatmung zu. Hierbei gilt es ein interdisziplinäres Konzept zu erstellen, das den individuellen Anforderungen jedes Patienten gerecht wird. Die Etablierung einer außerklinischen, nicht invasiven Beatmung ist ebenso sorgfältig zu planen und bedarf eines durchdachten Vorgehens bei der Entlassung. Aufgrund des jedoch deutlich höheren Aufwands bei einer invasiven Beatmung steht diese im Mittelpunkt dieses Artikels. Er soll eine Übersicht der wichtigsten Aspekte einer strukturierten Überleitung in die außerklinische invasive Beatmung bieten.

Das Weaningpotenzial sowie die Entscheidung zur Durchführung einer außerklinischen Beatmung sollte ein spezialisiertes Beatmungs- oder Weaningzentrum treffen. 


\section{Tabelle 1}

Untergruppen des prolongierten Weanings nach Definition der $\mathbf{S} \mathbf{k}$ Leitlinie „Prolongiertes Weaning“ (NIV: „non invasive ventilation“) [7].

\begin{tabular}{|c|c|c|}
\hline Gruppe & Kategorie & Definition \\
\hline $3 a$ & $\begin{array}{l}\text { prolongiertes } \\
\text { Weaning ohne } \\
\text { NIV }\end{array}$ & $\begin{array}{l}\text { erfolgreiche Entwöhnung mit Extubation/Dekanü- } \\
\text { lierung erst nach mindestens } 3 \text { erfolglosen Spon- } \\
\text { tanatmungsversuchen oder Beatmung länger als } \\
7 \text { Tage nach dem ersten erfolglosen Spontan- } \\
\text { atmungsversuch ohne Zuhilfenahme der NIV }\end{array}$ \\
\hline $3 b$ & $\begin{array}{l}\text { prolongiertes } \\
\text { Weaning mit } \\
\text { NIV }\end{array}$ & $\begin{array}{l}\text { erfolgreiche Entwöhnung mit Extubation/Dekanü- } \\
\text { lierung erst nach mindestens } 3 \text { erfolglosen Spon- } \\
\text { tanatmungsversuchen oder Beatmung länger als } \\
7 \text { Tage nach dem ersten erfolglosen Spontan- } \\
\text { atmungsversuch und nur mit Einsatz der NIV, ggf. } \\
\text { mit Fortsetzung der NIV als außerklinische Beat- } \\
\text { mung (weitere Differenzierung siehe Text) }\end{array}$ \\
\hline $3 c$ & $\begin{array}{l}\text { erfolgloses } \\
\text { Weaning }\end{array}$ & $\begin{array}{l}\text { Tod oder Entlassung mit invasiver Beatmung über } \\
\text { Tracheostoma }\end{array}$ \\
\hline
\end{tabular}

\section{Patientenauswahl}

Weaningkategorien. Die invasive Beatmung ist bei Patienten mit akuter respiratorischer oder ventilatorischer Insuffizienz eine lebenserhaltende Akuttherapie. Insbesondere bei multimorbiden und pneumologisch vorerkrankten Patienten muss jedoch häufig die Beatmung fortgesetzt werden, auch wenn die ursprüngliche, zur Beatmung führende Erkrankung bereits behandelt ist. Etwa $50 \%$ der gesamten Beatmungsdauer beansprucht die Entwöhnung von der maschinellen Beatmung (Weaning) [5,6]. Das Weaning wird in 3 Kategorien unterteilt [7]:

- Gruppe 1: einfaches Weaning

- Gruppe 2: schwieriges Weaning

- Gruppe 3: prolongiertes Weaning

Vom prolongierten Weaning spricht man bei Patienten, bei denen die Entwöhnung erst nach 3 Spontanatmungsversuchen oder nach über 7 Tagen nach dem ersten Spontanatmungsversuch gelingt. Das Entwöhnungsversagen ist definiert als:

- gescheiterter Spontanatmungsversuch

- Reintubation oder Rekanülierung und/oder Wiederaufnahme der maschinellen Unterstützung

- Tod innerhalb von 48 Stunden nach Extubation

Eine nicht invasive Beatmung zur Vermeidung einer invasiven Beatmung sollte man bei jedem Patienten in Betracht ziehen [8].
In einer aktuellen multizentrischen Studie wurden intensivmedizinisch betreute Patienten untersucht, bei denen nach invasiver Beatmung der Weaningprozess eingeleitet wurde. 14\% der Patienten wurden der Gruppe des prolongierten Weanings zugeordnet [9], von denen wiederum $74 \%$ erfolgreich entwöhnt werden konnten. Das Kollektiv der Patienten, die sich im prolongierten Weaning befinden, präsentiert sich hinsichtlich ihrer relevanten Erkrankungen sowie der weiteren Beatmungsdauer und Prognose sehr heterogen. Daher unterscheidet die interdisziplinäre S2kLeitlinie zum prolongierten Weaning in der Gruppe 3 die folgenden Untergruppen (Tab. 1) [7]:

- Gruppe 3a: prolongiertes Weaning ohne nicht invasive Beatmung

- Gruppe 3b: prolongiertes Weaning mit nicht invasiver Beatmung

- Gruppe 3c: erfolgloses Weaning

Die interdisziplinäre Behandlung und Evaluation des Weaningpotenzials dieser komplex kranken Patienten sollte möglichst ein spezialisiertes Weaningzentrum vornehmen. Die zertifizierten Weaningzentren sind unter der Schirmherrschaft der Deutschen Gesellschaft für Pneumologie und Beatmungsmedizin (DGP) in einem Kompetenznetzwerk, dem WeanNet, verbunden. Ziel ist hierbei die Aufnahme aller Weaningpatienten in ein Register, verbunden mit einer Qualitätsstandardisierung und Qualitätssicherung der Weaningzentren (www.pneumologie.de).

Voraussetzungen. Im Weaningzentrum sollte vor einer Überleitung in die außerklinische Beatmung überprüft werden, dass ein Weaningfortschritt innerhalb der kommenden 4 Wochen nicht zu erzielen ist (Infobox 1 ) [7]. Zudem sollte die vollständige klinische Diagnostik

\section{Infobox 1}

Medizinische Voraussetzungen zur Entlassung in die invasive außerklinische Beatmung

- Möglichkeit zur Umstellung auf eine nicht invasive Beatmung wurde überprüft

- keine Fortschritte im Weaningprozess innerhalb der nächsten 4 Wochen zu erwarten

- stabile Beatmungs- und Organfunktion seit mindestens 7 Tagen

- stabiler Beatmungszugang (meist plastisches Tracheostoma)

- hämodynamisch stabil ohne kreislaufunterstützende Medikamente

- gesicherte Ernährung (oral oder über Magensonde) 
abgeschlossen sowie eine außerklinisch durchführbare Dauertherapie eingeleitet sein. Der Patient und dessen Angehörige sollten in ausführlichen Gesprächen über die Tragweite und Risiken einer außerklinischen Beatmung aufgeklärt werden. Entscheidend ist auch ein vom Patient bzw. dessen Betreuer schriftlich dokumentierter Wunsch zur Durchführung einer außerklinischen invasiven Beatmung.

Indikation. Die Indikation zur Einleitung einer außerklinischen Beatmung wurden in der S2-Leitlinie der DGP definiert [10] und schließt vor allem Patienten mit thorakorestriktiven und neuromuskulären Erkrankungen sowie mit Obesitas-Hypoventilations-Syndrom (OHS) und chronisch obstruktiver Lungenerkrankung (COPD) ein. Bis auf die Patienten mit COPD liegen gute Daten hinsichtlich einer Verbesserung der Lebensqualität und des Langzeitüberlebens durch eine Langzeitbeatmung vor [10]. Zudem wurde von der DGP ergänzend ein Positionspapier mit Durchführungsempfehlungen zur invasiven außerklinischen Beatmung verfasst [11].

\section{Überleitung in die außerklinische Beatmung}

\section{Planung und Organisation}

Überleitteam. Die Planung und Organisation einer außerklinischen invasiven Beatmung bedarf eines interdisziplinären Überleitteams, bestehend aus Ärzten, Pflegeteam, Therapeuten sowie dem Sozialdienst. Die beteiligten Personen sollten einen erfahrenen Fallmanager bestimmen, der die Koordination der beteiligten Personen und Institutionen übernimmt und im Optimalfall eine Überleitkonferenz mit Anwesenheit aller beteiligten Personen einberuft. Die Hauptaufgabe vor der Entlassung besteht im Aufstellen und Einleiten einer an die Bedürfnisse des Patienten angepassten Therapie, die aus dem stationären Bereich in das außerklinische Umfeld überführt werden muss. Die Therapie muss umso umfassender sein, je abhängiger der Patient vom Beatmungsgerät und je eigeschränkter seine Eigenständigkeit ist. Von entscheidender Bedeutung ist zudem die Auswahl des außerklinischen Umfeldes (Abb. 1).

Überleitprozess. Mit dem Überleitprozess sollte man spätestens 2 Wochen vor dem geplanten Entlassungszeitpunkt beginnen [11]. In dieser Zeit muss die weitere ärztliche und pflegerische Versorgung sichergestellt

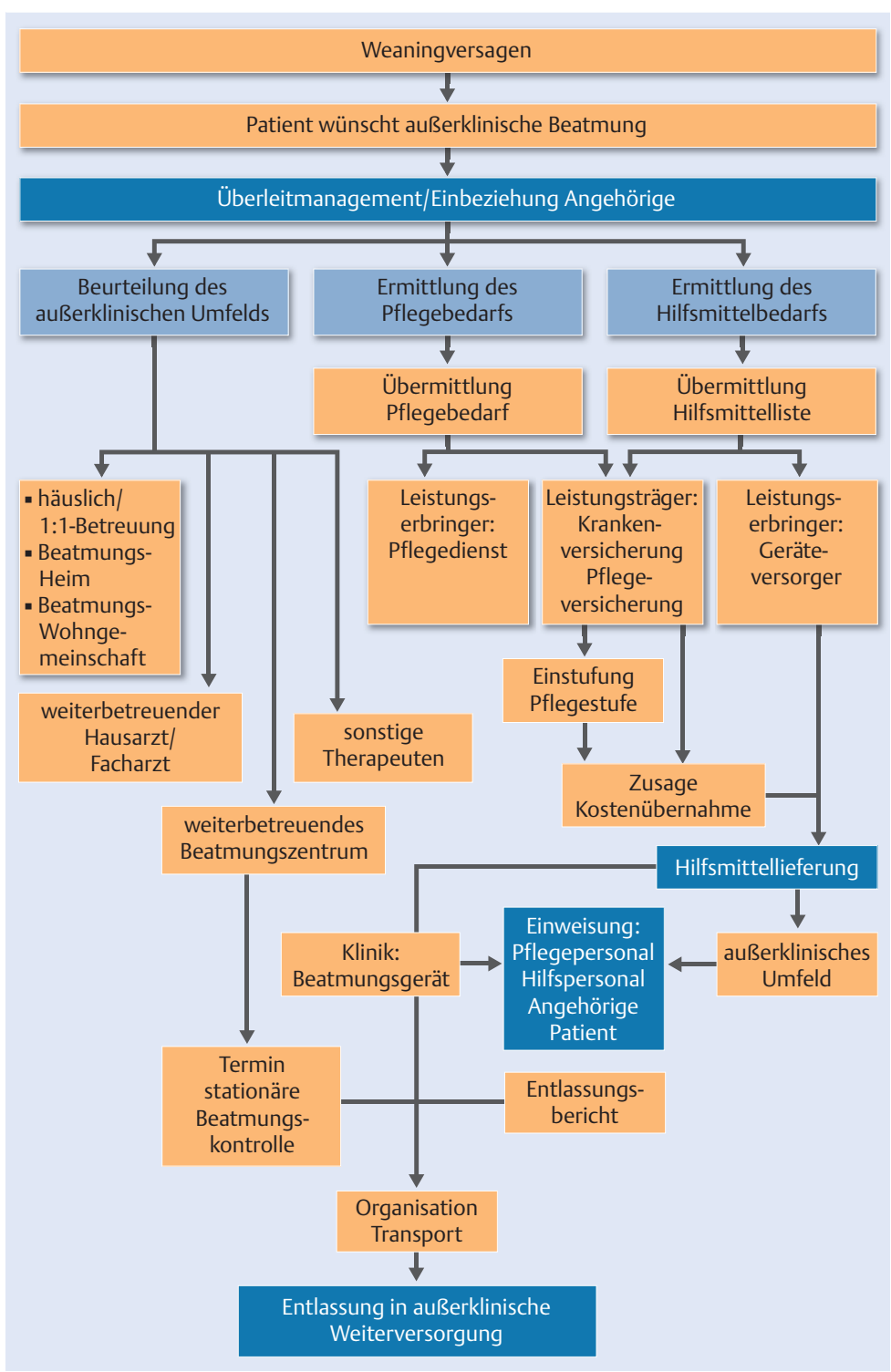

Abb. 1 Ablaufplan der Überleitung aus der Klinik in die außerklinische invasive Beatmung.

werden. Hierzu wird in der Regel eine Pflegestufe beim Leistungsträger (Krankenversicherung, Pflegeversicherung) beantragt, der über den Medizinischen Dienst der Krankenkassen die Einstufung vornimmt (Pflegestufe 1-3). Zudem erstellt man eine Liste aller notwendigen Hilfsmittel und übermittelt sie an den Leistungsträger und an den Leistungserbringer (Geräteversorger) [12]. Vor der Entlassung muss die Kostenzusage des Leistungsträgers vorliegen. Abschließend überprüft man die Vollständigkeit der Hilfsmittel in der weiterführenden Einrichtung bzw. im Krankenhaus. Verlegt wird der invasiv beatmete Patient mit einem Intensivtransport, der spätestens einen Tag vor der Entlassung organisiert werden muss. 
Das Überleitmanagement zur außerklinischen invasiven Beatmung sollte spätestens 2 Wochen vor dem geplanten Entlassungszeitpunkt beginnen.

\section{Hilfsmittelversorgung}

Vor der Entlassung aus dem Krankenhaus ist eine gründliche und individuelle Abklärung des Bedarfs an Hilfsmitteln erforderlich (Infobox 2) [8], am besten anhand von Checklisten. Die Verantwortung für die Zusammenstellung trägt der behandelnde Arzt. Die sachgerechte Benutzung und die Anwendungsintervalle der einzelnen Maßnahmen bespricht man einerseits bei mündlichen oder praktischen Übergaben, andererseits nimmt man sie auch in den pflegerischen und ärztlichen Bericht auf. Im folgenden Abschnitt werden die wichtigsten Hilfsmittel in Kürze vorgestellt.

\section{Infobox 2}

Hilfsmittelversorgung für die außerklinische invasive Beatmung

- Beatmung

- Sauerstoffversorgung

- Atemgaskonditionierung

- Inhalation

- Absaugen

- Überwachung

- pflegerische Hilfsmittel

Beatmung. Für jeden Patienten mit chronischer Ateminsuffizienz ist die Beatmung lebenserhaltend. Entscheidender Baustein der Beatmungstherapie ist das Beatmungsgerät. Die Auswahl des Geräts und die Wahl der Beatmungseinstellungen liegen in der Verantwortung des behandelnden Arztes, der diese Parameter im stationären Umfeld beurteilt und in den Entlassungspapieren dokumentiert. Die Beatmungs- und Spontanatmungszeiten müssen eindeutig definiert sein. Zudem ist für Patienten mit einer täglichen Beatmungszeit von mehr als 16 Stunden die Verordnung eines identischen Zweitgeräts für den Notfall obligat [10]. Vor allem bei mobilen Patienten sollte man auf die Verwendung eines entsprechend portablen Beatmungsgeräts achten. Die Beatmungsgeräte müssen vor Entlassung ins Krankenhaus geliefert werden, wo man sie überprüft und den weiterbetreuenden Personen die Bedienung erklärt. Die Umstellung auf ein anderes Beatmungsgerät oder Veränderungen der Beatmungsparameter sollte man nur stationär in der Klinik vornehmen, wohingegen der Wechsel auf ein baugleiches Gerät durch den Geräteversorger außerklinisch möglich ist [10].

Die Beatmung wird bei der außerklinischen invasiven Beatmung über ein Tracheostoma durchgeführt. Hierzu sollte ein epithelialisiertes (plastisches) und damit stabiles Tracheostoma angelegt sein, da bei Dilatationstracheostomata das Risiko einer kurzfristigen Schrumpfung mit der Unmöglichkeit einer Re-Kanülierung oder auch einer Kanülenfehllage besteht [10].

Die Wahl der Trachealkanülen richtet sich nach der Größe des Tracheostomas. Um den Atemwegswiderstand so gering wie möglich zu halten, wählt man das größtmögliche Lumen [13]. Die weiteren Eigenschaften der Kanülen richten sich nach den Fähigkeiten und Bedürfnissen des Patienten und sollten auch den Absaugbedarf und eine erhaltene Sprechfähigkeit berücksichtigen [14]. Größe und Art der Kanülen sowie die Wechselintervalle (in der Regel alle 4 Wochen) sind sorgfältig im Verlegungsbericht zu dokumentieren. Für die Lebensqualität ist die Erhaltung der Kommunikation ein entscheidender Baustein [15]. Daher prüft man stets die Möglichkeit einer Versorgung mit Sprechventil und der Verwendung einer ungeblockten Kanüle unter Berücksichtigung des Gasaustauschs und der Aspirationsgefahr. Ist eine entsprechende Versorgung nicht möglich, sind weitere Kommunikationsmöglichkeiten wie Buchstabentafeln oder ein Sprachcomputer zu erwägen.

Sauerstoffversorgung. Die Indikation zur Langzeitsauerstofftherapie ist vor der Überleitung in die außerklinische Beatmung im Rahmen wiederholter Blutgasanalysen zu prüfen $[18,19]$. Bei den Sauerstoffgeräten unterscheidet man zwischen Konzentratoren, Flüssigsauerstoffbehältern und Sauerstoffdruckflaschen. Die Wahl des Geräts hängt ab von der Mobilität des Patienten und der erforderlichen Sauerstoffflussrate. Für die Verordnung ist der behandelnde Arzt verantwortlich. Diese muss mit genauen Angaben über den Sauerstoffbedarf (Flussrate in Liter pro Minute unter Spontanatmung, Beatmung und evtl. unter Belastung) und die täglichen Mobilitätsstunden an die Krankenkasse und den Versorger übermittelt werden. Der Versorger muss eine Lieferung innerhalb von 24 Stunden, die Einweisung in die Geräte und die reibungslose Weiterversorgung gewährleisten.

Atemgaskonditionierung. Der durch das Tracheostoma veränderte Atemweg erhöht das Risiko einer Austrocknung der Schleimhäute und einer Verdickung des 
Bronchialsekrets. Daher ist eine Atemgaskonditionierung mit passiver oder aktiver Atemgasbefeuchtung essenziell [16]. Bei invasiver Beatmung verwendet man in der Regel eine aktive Befeuchtung, da sich passive Systeme („heat and moisture exchanger“, HME) negativ auf die Beatmungsqualität auswirken können [30]. Zudem ist die Anpassung einer inhalativen Therapie zur Befeuchtung der Atemwege und die Applikation einer spezifischen antiobstruktiven oder antibiotischen Therapie mit einem geeigneten Inhalationssystem notwendig [17].

Pulsoxymetrie. Zur Überwachung der außerklinischen Beatmung dient die Pulsoxymetrie. Hierzu legt man individuell je nach Indikation die Kontrollintervalle fest. Eine Überwachung des Kohlendioxidpartialdrucks $\left(\mathrm{pCO}_{2}\right)$ ist in den meisten Fällen nicht indiziert [10].

Inhalation und Absaugen. Das Absaugen ist bei invasiv beatmeten Patienten essenzieller Bestandteil der täglichen Therapie mit einem hohen zeitlichen und personellen Aufwand. Die sorgfältige Anpassung an die Bedürfnisse des Patienten und die adäquate Anwendung im außerklinischen Umfeld reduzieren die Rate pulmonaler Infekte und Sekretverhalte. Dadurch vermeidet man auch Hypoxämien und damit verbundene wiederholte Hospitalisierungen [20].

Neben der Basisversorgung, bestehend aus Absauggerät, Atemgasbefeuchtung und Inhalationsgerät, ist vor allem bei Patienten mit neuromuskulären Erkrankungen die Notwendigkeit einer maschinellen Hustenassistenz zu prüfen [21]. Hierfür ist die Datenlage bisher jedoch eingeschränkt, weshalb eine explizite Anwendungsbegründung dem Antrag zur Kostenübernahme beigefügt werden muss. Für die Maßnahmen und Anwendungsintervalle des Absaugens ist neben einem Protokoll auch eine detaillierte Einweisung des Pflegepersonals erforderlich.

Pflegerische Hilfsmittel. Neben den alltäglichen Verbrauchsmaterialien sind je nach Bedürfnissen des Patienten unterschiedliche pflegerische Hilfsmittel wie Rollstuhl, Pflegebett, Toilettenstuhl, Hebevorrichtungen oder Kommunikationshilfen erforderlich. Unter Umständen muss man auch eine Ernährung über die Magensonde organisieren. Der Umfang richtet sich dabei auch nach dem außerklinischen Umfeld. Pflegerische Hilfsmittel werden anders als die beatmungsassoziierten Hilfsmittel bei der Pflegekasse beantragt und bedürfen unterschiedlich hoher privater Zuzahlungen.
Die sorgfältige Ermittlung und rechtzeitige Verordnung der notwendigen Hilfsmittel ist die Basis der außerklinischen invasiven Beatmung.

\section{Außerklinisches Umfeld}

Die Beatmungstherapie wird mit dem Zeitpunkt der Entlassung aus der Klinik zur außerklinischen Beatmung. Dabei ist die Möglichkeit einer häuslichen Versorgung, die personell aufwendig und teuer ist, keinesfalls selbstverständlich. 1981 wurde in den USA der Fall Katie Backett zu einem Präzedenzfall, der den gesetzlichen Anspruch auf eine häusliche Weiterversorgung beatmeter Patienten und damit die bestmögliche Integration in die Gesellschaft zusichern sollte [22]. Ähnliches galt in Deutschland für das „DrachenfliegerUrteil“ von 1999, das spätestens nach einer Revision im Jahre 2010 die überwiegende Finanzierung der außerklinischen Beatmung über die Krankenkassen gewährleistet [23]. Die außerklinische Weiterversorgung soll zu einer Steigerung der Lebensqualität durch Eigenständigkeit und Eingliederung in das soziale Umfeld führen [24].

Die außerklinische Versorgung führt auch zu einer finanziellen Entlastung des Gesundheitssystems im Vergleich zu einer äquivalenten stationären

Behandlung [24].

Dennoch bedarf eine außerklinische Versorgung einer fachpflegerischen Betreuung auf hohem Niveau und ist dementsprechend auch teuer. Welche Behandlung nun im Endeffekt die günstigere Form darstellt, ist letztlich für jedes Land nicht genau bekannt und hängt sowohl vom individuellen Fall als auch vom Gesundheitssystem selbst ab.

Die betreuenden Pflegedienste müssen den von der Deutschen interdisziplinären Gesellschaft für außerklinische Beatmung (DIGAB) e.V. definierten Standards genügen [10]. Unabhängig vom Ort der Unterbringung muss die Versorgung durch ein spezialisiertes Pflegeteam gewährleistet sein, das vor allem Kompetenzen hinsichtlich der Beatmung und Notfallsituationen aufweisen muss. Die Betreuung ist entweder im häuslichen Umfeld oder in Beatmungspflegeheimen bzw. Beatmungswohngemeinschaften möglich.

Die Wahl der Unterbringung ist neben den medizinischen Aspekten unter Berücksichtigung von psychologischen, sozialen, finanziellen und geografischen Gesichtspunkten zu treffen und sollte in erster Linie 
den Wünschen des Patienten und den familiären Ressourcen entsprechen.

Die außerklinische Weiterversorgung ist im häuslichen Umfeld oder in spezialisierten Beatmungspflegeheimen und Beatmungswohngemeinschaften möglich.

\section{Häusliche Weiterversorgung}

Für die häusliche Weiterversorgung müssen sowohl ein passendes soziales Umfeld als auch bauliche Voraussetzungen gegeben sein. Unter Umständen sind vor der Entlassung Umbaumaßnahmen für eine pflegegerechte Versorgung im häuslichen Umfeld notwendig. Die fachpflegerische Versorgung des Patienten wird von einem beauftragten Pflegedienst in einer 1:1-Betreuung rund um die Uhr übernommen, der von Pflegekräften mit geringerem Qualifikationsniveau unterstützt wird. Der Pflegedienst beurteilt die Tauglichkeit des häuslichen Umfeldes zur außerklinischen Beatmung und kann im Einzelfall eine Betreuung unter den gegebenen Umständen auch ablehnen.

Aufgrund finanzieller und sozialer Aspekte wird die Einbeziehung von Laienhelfern (z. B. Angehörige) in die Pflege empfohlen. Diese sollen in die grundpflegerischen Maßnahmen eingewiesen werden und diese mit der Zeit möglichst im Sinne einer Rückzugspflege vollständig übernehmen [15]. Dabei ist eine größtmögliche Autonomie der Familie des Patienten erstrebenswert. Sollte eine häusliche Versorgung nicht möglich oder nicht erwünscht sein, stehen spezialisierte Beatmungseinrichtungen zur Verfügung.

Beatmungspflegeheim. Beatmungspflegeheime sind vollstationäre Pflegeeinrichtungen, die sich auf die Betreuung beatmeter Patienten spezialisiert haben. Je nach Pflegebedarf und Pflegestufe können hier unterschiedlich hohe private Zuzahlungen anfallen.

Beatmungswohngemeinschaften. In einer Beatmungswohngemeinschaft mietet der Patient auf eigene Kosten ein Zimmer in einer Wohngemeinschaft, in der weitere Patienten mit außerklinischer Beatmung versorgt werden. Jeder Patient hat die theoretisch freie Wahl eines geeigneten fachspezifischen Pflegedienstes, wobei in der alltäglichen Praxis die Versorgung aller Bewohner einer Wohngemeinschaft meist über denselben Pflegedienst geregelt ist.

\section{Lebensqualität bei invasiver außerklinischer Beatmung}

Neben der medizinischen Versorgung ist in den letzten Jahren die gesundheitsbezogene Lebensqualität in den Fokus der außerklinischen Versorgung gerückt. Diese beinhaltet ein multidimensionales Konstrukt, dass das subjektive Befinden auf psychischer, physischer und sozialer Ebene beschreibt. Die Lebensqualität wird zunehmend als Therapieziel definiert, die bei Patienten mit invasiver Beatmung einen der Überlebensdauer gleichzusetzenden Stellenwert erhält. Dabei wird die Lebensqualität überwiegend über Fragebögen erhoben, die entweder krankheitsübergreifend oder krankheitsspezifisch ausgerichtet sind. Der „Severe Respiratory Insufficiency Questionnaire“ ist der einzige krankheitsspezifische Fragebogen, der bisher bei Patienten mit invasiver Beatmung eingesetzt wurde [25,26]. Der Fragebogen ist inzwischen in 9 Sprachen übersetzt worden und über die Internetseite der DGP kostenlos verfügbar (www.pneumologie.de).

Bisher besteht eine eingeschränkte Datenlage zur gesundheitsbezogenen Lebensqualität bei invasiver außerklinischer Beatmung. Die Studien untersuchten überwiegend Patienten mit neuromuskulären oder thorakal restriktiven Erkrankungen anhand standardisierter Fragebögen und qualitativer Beschreibungen [15]. Dabei scheint die Unterscheidung zwischen invasivem und nicht invasivem Beatmungszugang einen nur geringen Einfluss auf die Lebensqualität zu haben $[27,28]$. Auch die Art der Unterbringung - im häuslichen Umfeld oder in einer spezialisierten Beatmungseinrichtung - hatte keine Auswirkungen auf die Lebensqualität [15]. Jedoch scheinen Patienten mit neuromuskulären Erkrankungen sowie erhaltener sozialer Integrität gegenüber Lungenerkrankten und isoliert lebenden Patienten eine höhere Lebensqualität unter invasiver Beatmung aufzuweisen.

Die Erhaltung der Lebensqualität des Patienten sollte in den Planungen der außerklinischen Beatmung in besonderem Maße berücksichtigt werden.

\section{Ärztliche außerklinische Betreuung}

Die ambulante ärztliche Weiterbetreuung beatmeter Patienten stellt einen Engpass im deutschen Gesundheitssystem dar. Sie gestaltet sich nicht zuletzt durch die bestehende strikte Trennung von ambulanter und 
stationärer sowie hausärztlicher und fachärztlicher Betreuung schwierig [29].

Der weiterbehandelnde Arzt sollte besondere Kenntnisse im Bereich der Beatmung aufweisen. Derzeit wird der Großteil der beatmeten Patienten über den Hausarzt weiterversorgt, obwohl dieser nur selten eine entsprechende Expertise nachweisen kann. Daher ist es wünschenswert, dass je nach Grunderkrankung der niedergelassene Pneumologe, Anästhesist oder Neurologe in die Betreuung einbezogen wird [10]. Dieser ist rechtzeitig über die anstehende Entlassung in die außerklinische Beatmung, den aktuellen Gesundheitszustand und den zu erwartenden Krankheitsverlauf zu informieren. Aufgrund der häufigen Immobilität der Patienten sind in den meisten Fällen ärztliche Hausbesuche notwendig.

Die erste Kontrolle der außerklinischen Beatmung sollte 4-8 Wochen nach der Entlassung stationär im betreuenden Beatmungszentrum stattfinden.

\section{Beatmungszentrum}

Laut der bestehenden Leitlinien sollte jeder Patient mit außerklinischer Beatmung an ein Beatmungszentrum angebunden sein [10]. In einer bundesweiten Umfrage unter den Pflegediensten zeigte sich, dass lediglich 43\% der Patienten von einem Beatmungs- oder Weaningzentrum betreut wurden [29]. In Anbetracht der beschriebenen ambulanten ärztlichen Versorgung scheint eine adäquate beatmungsspezifische Betreuung bei diesen Patienten unmöglich.

Die außerklinische Beatmung sollte in regelmäßigen Abständen in einem Beatmungszentrum kontrolliert werden. Die Anfangsphase der außerklinischen Versorgung ist besonders kritisch. Daher wird eine erste Wiedervorstellung nach 4-8 Wochen vorgesehen und bereits bei der Entlassung vereinbart [10]. Weitere Kontrollen folgen dann 1- bis 2-mal jährlich, bei eingeschränkter Therapietreue auch in kürzeren Intervallen. Bei jeder Wiedervorstellung sollte man das aktuelle Weaningpotenzial überprüfen. Eine außerklinische Entwöhnung von der Beatmung ist in der Leitlinie nicht vorgesehen und sollte nicht durchgeführt werden $[10,11]$. Bei klinischer Verschlechterung des Patienten muss im Idealfall die Wiederaufnahme in das betreuende Beatmungszentrum jederzeit möglich sein. Alternativ kann man im Vorfeld ein weiteres Krankenhaus für eine mögliche Akutversorgung einbeziehen.

\section{Kernaussagen}

- Die Beurteilung des Weaningpotenzials sowie die Entscheidung zur Durchführung einer außerklinischen Beatmung sollte ein spezialisiertes Beatmungs- bzw. Weaningzentrum vornehmen.

- Die Planung und Vorbereitung einer außerklinischen invasiven Beatmung sollte spätestens 2 Wochen vor dem geplanten Entlassungszeitpunkt begonnen werden.

- Die sorgfältige Ermittlung und rechtzeitige Verordnung der notwendigen Hilfsmittel ist die Basis der außerklinischen invasiven Beatmung.

\section{Sonstige außerklinische Betreuung}

Im Rahmen der außerklinischen Betreuung ist die Einbeziehung weiterer spezialisierter Fachkräfte wichtig. Hierzu zählen Atmungstherapeuten, Logopäden, Physiotherapeuten, Ergotherapeuten, Pädagogen und Sozialpädagogen. Deren Einsatz sollten individuell den Bedürfnissen des Patienten angepasst und bereits vor Entlassung organisiert werden.

Interessenkonflikt: Die Autoren geben an, dass sie Forschungsgelder für wissenschaftliche Studien sowie Vortragshonorare und Kongressunterstützungen von Firmen erhalten haben, die Beatmungsgeräte herstellen oder vertreiben.

\section{Über die Autoren}

\section{Jens Callegari}

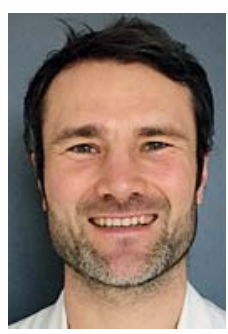

Dr. med. Jahrgang 1981. 2002-2009

Studium der Humanmedizin an der Ludwig-Maximilians-Universität und Technischen Universität München. 2010-2012 Grundlagenforschung mit Schwerpunkt Lungenemphysem am Comprehensive Pneumology Centre in München. 2012-2013 Assistenzarzt in der Abteilung für Pneumologie im Krankenhaus Neuwittelsbach in München. Seit 2013 Assistenzarzt und wissenschaftlicher Mitarbeiter in der Abteilung für Pneumologie der Lungenklinik Köln-Merheim. 


\section{Wolfram Windisch}

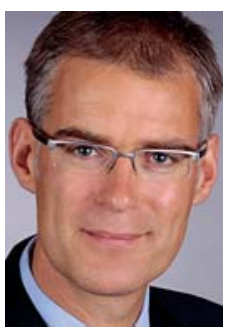

Prof. Dr. med. Jahrgang 1969. 1990-1996 Studium der Humanmedizin an der Georg-AugustUniversität in Göttingen. 1997-2003 Facharztausbildung für Innere Medizin in der Abteilung für Pneumologie der Universitätsklinik Freiburg. 2004-2011 Oberarzt der Abteilung für Pneumologie der Universitätsklinik Freiburg. 2007-2011 Facharztanerkennung für Pneumologie, Intensivmedizin und Allergologie an der Universitätsklinik Freiburg. Seit 2011 Chefarzt der Abteilung für Pneumologie der Lungenklinik Köln-Merheim. Seit 2014 Lehrstuhlinhaber des Bereichs Pneumologie der Universität Witten/Herdecke. Federführender Autor der Leitlinie für außerklinische Beatmung und Koautor der Leitlinie „Prolongiertes Weaning“.

\section{Jan Hendrik Storre}

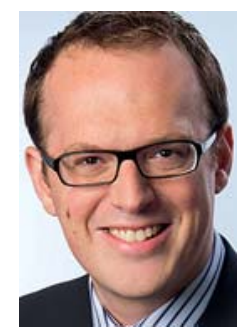

PD Dr. med. Jahrgang 1977. 1997-2004 Studium der Humanmedizin, Albert-Ludwigs-Universität Freiburg. 2005-2011 Facharztausbildung in der Abteilung Pneumologie und Medizinische Klinik an der Universitätsklinik Freiburg. Seit 2011 leitender Oberarzt der Abteilung für Pneumologie der Lungenklinik KölnMerheim. Facharzt für Innere Medizin, Pneumologie sowie Zusatzbezeichnung Schlafmedizin. Habilitation 2011 an der Universität Freiburg, Thema: Monitoring der Ventilation bei nicht invasiver Beatmung. Seit 2013 im Vorstand der DIGAB sowie seit 2014 Fachexperte für das Institut für Lungenforschung (ILF) zur Zertifizierung von Weaningzentren der DGP.

\section{Korrespondenzadresse}

Dr. med. Jens Callegari

Lungenklinik Köln-Merheim

Kliniken der Stadt Köln gGmbH

Lehrkrankenhaus der Universität Witten/Herdecke

Ostmerheimer Str. 200

51109 Köln

E-Mail: callegarij@kliniken-koeln.de

\section{Literatur}

1 Nasiłowski J, Wachulski M, Trznadel W et al. The evolution of home mechanical ventilation in poland between 2000 and 2010. Respir Care 2015; 60: 577 - 585

2 Lloyd-Owen SJ, Donaldson GC, Ambrosino N et al. Patterns of home mechanical ventilation use in Europe: results from the Eurovent survey. Eur Respir J 2005; 25: 1025 - 1031

3 Garner DJ, Berlowitz DJ, Douglas J et al. Home mechanical ventilation in Australia and New Zealand. Eur Respir J 2013; 41: $39-45$

4 Rose L, McKim DA, Katz SL et al. Home mechanical ventilation in Canada: a national survey. Respir Care 2015; 60: 695 - 704

5 Esteban A, Anzueto A, Frutos F et al. Mechanical Ventilation International Study Group. Characteristics and outcomes in adult patients receiving mechanical ventilation: a 28-day international study. JAMA 2002; 287: 345 - 355

6 Ely EW, Baker AM, Dunagan DP et al. Effect on the duration of mechanical ventilation of identifying patients capable of breathing spontaneously. N Engl J Med 1996; 335: 1864 1869

7 Schönhofer B, Geiseler J, Dellweg D et al. Prolonged weaning: S2k-guideline published by the German Respiratory Society. Pneumologie 2014; 68: 19-75

8 Schönhofer B, Euteneuer S, Nava S et al. Survival of mechanically ventilated patients admitted to a specialised weaning centre. Intensive Care Med 2002; 28: 908-916

9 Funk GC, Anders S, Breyer MK et al. Incidence and outcome of weaning from mechanical ventilation according to new categories. Eur Respir J 2010; 35: 88-94

10 Windisch W, Walterspacher S, Siemon K et al. Guidelines for non-invasive and invasive mechanical ventilation for treatment of chronic respiratory failure. Published by the German Society for Pneumology (DGP). Pneumologie 2010; 64: 640 652

11 Randerath WJ, Kamps N, Brambring J et al. Recommendations for invasive home mechanical ventilation. Pneumologie 2011; 65: $72-88$

12 Callegari J, Storre JH. Medical and technical aid for patients receiving home mechanical ventilation. In: Themenheft außerklinische Beatmung. Bad Lippspringe: Atemwegsliga; 2015

13 Oczenski W. Atmen - Atemhilfen: Atemphysiologie und Beatmungstechnik. Stuttgart: Thieme; 2012

14 Bach JR, Alba AS. Tracheostomy ventilation. A study of efficacy with deflated cuffs and cuffless tubes. Chest 1990; 97 : $679-683$

15 Huttmann SE, Windisch W, Storre JH. Invasive Home Mechanical Ventilation: Living Conditions and Health-related Quality of Life. Respiration 2015; 89: $312-321$

16 Nakagawa NK, Macchione M, Petrolino HM et al. Effects of a heat and moisture exchanger and a heated humidifier on respiratory mucus in patients undergoing mechanical ventilation. Crit Care Med 2000; 28: 312-317

17 Kallet RH. Adjunct therapies during mechanical ventilation: airway clearance techniques, therapeutic aerosols, and gases. Respir Care 2013; 58: 1053-1073 
18 Magnussen H, Kirsten AM, Köhler D et al. Deutsche Gesellschaft Für Pneumologie und Beatmungsmedizin e.V. Guidelines for long-term oxygen therapy. German Society for Pneumology and Respiratory Medicine. Pneumologie 2008; 62: $748-756$

19 Weitzenblum E, Sautegeau A, Ehrhart M et al. Long-term oxygen therapy can reverse the progression of pulmonary hypertension in patients with chronic obstructive pulmonary disease. Am Rev Respir Dis 1985; 131: 493- 508

20 Bach JR. Management of patients with neuromuscular disease. Philadelphia: Hanley \& Belfus; 1990

21 Geiseler J, Karg O. Management of secretion in patients with neuromuscular diseases. Pneumologie 2008; 62 : 0143-48

22 Shapiro J. Katie Beckett: Patient Turned Home-Care Advocate. Im Internet: http://www.npr.org/templates/story/story.php? storyld $=131145687$

23 BSG. Urteil vom 17.06.2010 - B 3 KR 7/09 R (lexetius.com/ 2010, 3629).

24 King AC. Long-term home mechanical ventilation in the United States. Respir Care 2012; 57: 921 - 930
25 Windisch W, Budweiser S, Heinemann F et al. The Severe Respiratory Insufficiency Questionnaire was valid for COPD patients with severe chronic respiratory failure. J Clin Epidemiol 2008; 61: 848-853

26 Windisch W. Quality of life in home mechanical ventilation study group. Impact of home mechanical ventilation on health-related quality of life. Eur Respir J 2008; 32: 1328 1336

27 Pehrsson K, Olofson J, Larsson S et al. Quality of life of patients treated by home mechanical ventilation due to restrictive ventilatory disorders. Respir Med 1994; 88: 21 -26

28 Markström A, Sundell K, Lysdahl M et al. Quality-of-Life Evaluation of Patients With Neuromuscular and Skeletal Diseases Treated With Noninvasive and Invasive Home Mechanical Ventilation. Chest 2002; 122: 1695-1700

29 Dellweg D, Gerhard F, Hoehn E et al. Survey of nursing services with regard to mechanical ventilation at home. Pneumologie 2011; 65: 685-691

30 Rathgeber J. Devices used to humidify respired gases. Respir Care Clin N Am 2006; 12: 165-182 


\section{CME-Fragen}

\section{CME.thieme.de}

\section{CME-Teilnahme}

- Viel Erfolg bei Ihrer CME-Teilnahme unter http://cme.thieme.de

- Diese Fortbildungseinheit ist 12 Monate online für eine CME-Teilnahme verfügbar.

- Sollten Sie Fragen zur Online-Teilnahme haben, unter http://cme.thieme.de/hilfe finden Sie eine ausführliche Anleitung.

1

Welche Aussage zum Weaning ist richtig?
A Die Weaningkategorie 1 beschreibt ein schwieriges Weaning.

B Als Kategorie 3a wird ein prolongiertes Weaning mit der Notwendigkeit einer weiteren nicht invasiven Beatmung verstanden.

C Die Weaningkategorie $3 c$ beschreibt ein erfolgloses Weaning.

D Bei der Weaningkategorie 3 wird immer die Überleitung in die außerklinische Beatmung notwendig.

E Alle Antworten sind richtig.
2

Welche Aussage über die Voraussetzungen für eine außerklinische invasive Beatmung ist falsch?
A Der Patient muss seit mindestens 7 Tagen stabile Organfunktionen aufweisen.

B Die Möglichkeit einer nicht invasiven Beatmung sollte geprüft sein.

C Der Patient/Betreuer muss einer außerklinischen invasiven Beatmung schriftlich zustimmen.

D Ein Weaningpotenzial ist in den kommenden 4 Wochen nicht zu erwarten.

E Aus pflegerischen Gründen ist ein dilatatives Tracheostoma (Punktions-Tracheostoma) einem plastischen Tracheostoma vorzuziehen.

\section{3}

Welche Aussage zur Hilfsmittelversorgung ist falsch?
A Bei Patienten mit einer täglichen Beatmungszeit von mehr als 16 Stunden ist die Verordnung eines identischen Zweitgeräts für den Notfall obligat.

B Die Umstellung auf ein anderes Beatmungsgerät oder Veränderungen der Beatmungsparameter sollte man nur stationär durchführen.

C Für die Zusammenstellung der Hilfsmittel ist der Sozialdienst verantwortlich.

D Für jeden Patienten ist die außerklinische Versorgung mit einem Absauggerät notwendig.

E Die Verantwortung für die Zusammenstellung der Hilfsmittel trägt der stationär behandelnde Arzt.

\section{4}

Welche Aussage zur außerklinischen Weiterversorgung der Patienten ist falsch?
A Sie ist finanziell und personell mit einem hohen Aufwand verbunden.

B Sie schließt möglichst auch die Angehörigen des Patienten ein.

C Sie kann sowohl in Beatmungspflegeheimen, Beatmungswohngemeinschaften, als auch im häuslichen Umfeld des Patienten stattfinden.

D Sie bedeutet pflegerisch in der Regel eine 2:1-Betreuung.

E Sie muss im Rahmen der Planung und Koordination der Überleitung rechtzeitig organisiert werden.

\section{5}

Welche Aussage zur invasiven außerklinischen Beatmung ist richtig?
A Die Überwachung der außerklinischen Beatmung übernimmt ausschließlich das Weaningzentrum.

B Der Überwachung des außerklinisch beatmeten Patienten dienen primär Blutgasanalysen.

C Die außerklinische Überwachung des Kohlendioxidpartialdrucks ist in den meisten Fällen indiziert.

D Die außerklinische Überwachung des Kohlendioxidpartialdrucks ist bei allen Patienten mit mehr als 16 Beatmungsstunden pro Tag notwendig.

E Die regelmäßige Kontrolle der Sauerstoffsättigung ist Bestandteil der außerklinischen Überwachung. 


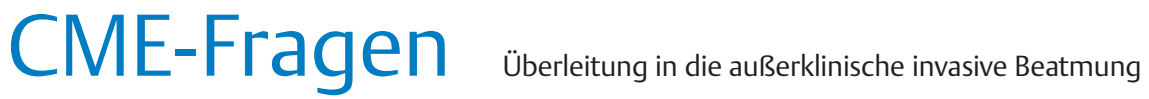

\section{6}

Welche Aussage über Beatmungsund Weaningzentren ist falsch?
A Die Entscheidung zur Durchführung und die Planung einer außerklinischen Beatmung sollte ein Weaningzentrum vornehmen.

B Die Beatmung sollte in regelmäßigen Abständen in dem betreuenden Beatmungszentrum stattfinden.

C Das Weaningpotenzial muss nach der Entlassung in die außerklinische Beatmung nicht mehr kontrolliert werden.

D Die erste Kontrolle der Beatmung sollte 4-8 Wochen nach Entlassung im betreuenden Beatmungszentrum stattfinden.

E Die Beatmungskontrollen führt man im Rahmen stationärer Aufenthalte im Beatmungszentrum durch.
7

Welche Aussage zur Lebensqualität ist richtig?
A Eine verbale Kommunikation ist bei einem tracheotomierten Patienten nicht möglich.
B Die Kommunikationsfähigkeit ist wichtig für die Erhaltung der Lebensqualität.
C Die Grunderkrankung hat keinen Einfluss auf die Lebensqualität des Patienten.
D Die Art der Unterbringung hat entscheidenden Einfluss auf die Lebensqualität.
E Alle Aussagen sind richtig.

8

Was zählt zu den Hilfsmitteln für den Umgang mit Sekret?

9

Welche Aussage ist falsch?
A Absauggerät
B Inhalationsgerät
C maschinelle Hustenassistenz
D Atemgasbefeuchtung
E Alle Aussagen sind richtig.
A Die Zusammenstellung der Hilfsmittel muss man frühzeitig an den Kostenträger übermitteln.
B Vor der Entlassung muss die Kostenübernahme vorliegen.
C Vor der Entlassung muss die Vollständigkeit der Hilfsmittel überprüft werden.
D Für die Wartung der technischen Hilfsmittel ist der Geräteversorger verantwortlich.
E Das Beatmungsgerät wird direkt ins weiterbetreuende außerklinische Umfeld geliefert.

\section{0}

Welche Aussage zur außerklinischen Beatmung ist richtig?
A Die Verteilung von invasiv und nicht invasiv beatmeten Patienten ist in allen Ländern gleich.

B Die Prävalenz außerklinisch beatmeter Patienten betrug 2001 in Europa 6,6 pro 100000 Einwohner.

C Der überwiegende Teil der außerklinisch beatmeten Patienten ist invasiv über ein Tracheostoma beatmet.

D In den letzten Jahren wurde eine Abnahme der außerklinisch beatmeten Patienten beobachtet.

E Alle Aussagen sind falsch. 